POST-PRINT VERSION

\title{
The role of the amygdala and the basal ganglia in visual processing of central vs. peripheral emotional content
}

\author{
Inês Almeida ${ }^{\mathrm{a}}$ \\ Visual Neuroscience Laboratory, IBILI, Faculty of Medicine, Coimbra, Portugal \\ Email:italmeida@fmed.uc.pt \\ Marieke van Asselen ${ }^{\mathrm{a}}$ \\ Visual Neuroscience Laboratory, IBILI, Faculty of Medicine, Coimbra, Portugal \\ Email:masselen@fmed.uc.pt
}

Miguel Castelo-Branco ${ }^{\mathrm{a}, \mathrm{b}}$ corresponding author

Visual Neuroscience Laboratory, IBILI, Faculty of Medicine, Coimbra, Portugal ICNAS, Brain Imaging Network of Portugal

Email:mcbranco@fmed.uc.pt

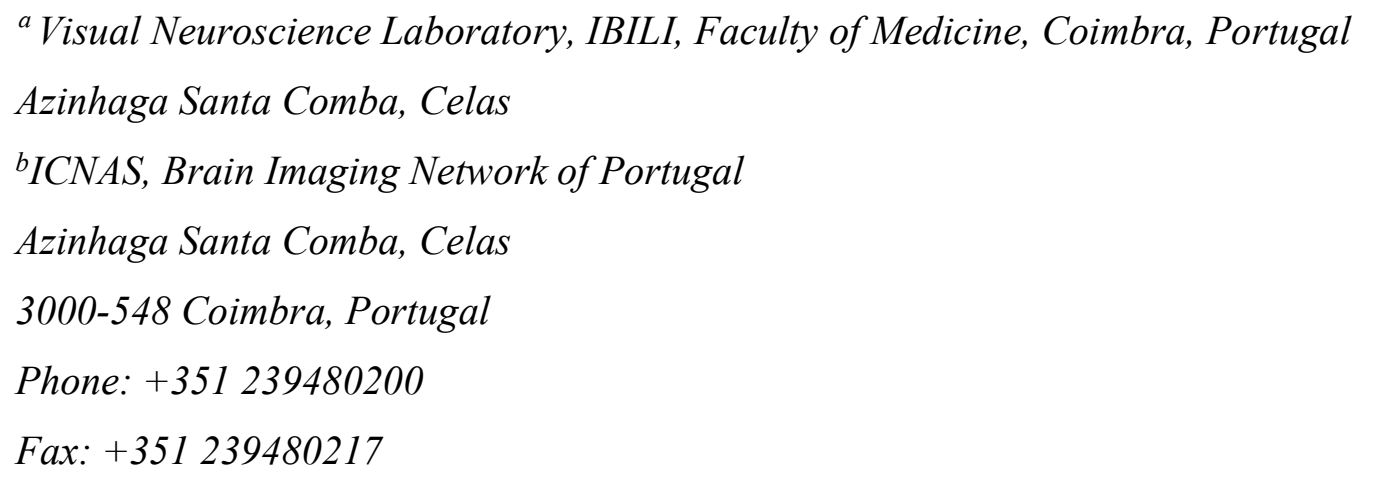




\begin{abstract}
In human cognition, most relevant stimuli, such as faces, are processed in central vision.

However, it is widely believed that recognition of relevant stimuli (e.g. threatening animal faces) at peripheral locations is also important due to their survival value. Moreover, task instructions have been shown to modulate brain regions involved in threat recognition (e.g. the amygdala). In this respect it is also controversial whether tasks requiring explicit focus on stimulus threat content vs. implicit processing differently engage primitive subcortical structures involved in emotional appraisal.
\end{abstract}

Here we have addressed the role of central vs. peripheral processing in the human amygdala using animal threatening vs. non-threatening face stimuli. First, a simple animal face recognition task with threatening and non-threatening animal faces, as well as non-face control stimuli, was employed in naïve subjects (implicit task). A subsequent task was then performed with the same stimulus categories (but different stimuli) in which subjects were told to explicitly detect threat signals.

We found lateralized amygdala responses both to the spatial location of stimuli and to the threatening content of faces depending on the task performed: the right amygdala showed increased responses to central compared to left presented stimuli specifically during the threat detection task, while the left amygdala was better prone to discriminate threatening faces from non-facial displays during the animal face recognition task. Additionally, the right amygdala responded to faces during the threat detection task but only when centrally presented. Moreover, we have found no evidence for superior responses of the amygdala to peripheral stimuli. Importantly, we have found that striatal regions activate differentially depending on peripheral 
vs. central processing of threatening faces. Accordingly, peripheral processing of these stimuli activated more strongly the putaminal region, while central processing engaged mainly the caudate nucleus.

We conclude that the human amygdala has a central bias for face stimuli, and that visual processing recruits different striatal regions, putaminal or caudate based, depending on the task and on whether peripheral or central visual processing is involved.

Keywords: amygdala, basal ganglia, implicit / explicit, central / peripheral, threat, faces. 


\section{Introduction}

Much of what we know regarding the functional anatomy of neural pathways connecting to the amygdala comes from auditory fear conditioning studies in the rat animal model (Whalen, Davis, Oler, Kim, Kim, \& Neta, 2009; LeDoux, J. \& Phelps, E., 2008). A large difference between rodents and primates can be recognized in the processing of social stimuli such as faces (Buchanan, Tranel \& Adolphs, 2009). In primate visual and affective processing, faces can be considered as a special class of objects (Critchley, Daly, Phillips, Brammer, Bullmore, Williams, et al., 2000; Hershler, Golan, Bentin, \& Hochstein, 2010; Johnson, 2005). Faces are preferentially processed in central vision, where they are screened for high-resolution foveal information (Kanwisher, 2001; Levy, Hasson, Avidan, Hendler, \& Malach, 2001). Studies in humans suggest the existence of foveally-biased specialized regions along the occipito-temporal ventral visual pathway to extract meaning from faces: the occipital gyrus, the lateral occipital (LO), the superior temporal sulcus (STS), and regions in the fusiform gyrus, such as the fusiform face area (FFA) (Grill-Spector, Knouf, \& Kanwisher, 2004; Kanwisher, McDermott, \& Chun, 1997). Accordingly, regions in the fusiform gyrus, such as within the FFA complex, are tuned to a broad category of faces (Tong, Nakayama, Moscovitch, Weinrib, \& Kanwisher, 2000), specially when these are presented in central vision, but they do nevertheless also respond to peripherally presented faces (Faivre, Charron, Roux, Lehéricy, \& Kouider, 2012; Morawetz, Baudewig, Treue, \& Dechent, 2010; Kanwisher, 2001).

Subcortical regions such as the amygdala are also involved in face meaning extraction (Atkinson, \& Adolphs, 2011; Gothard, Battaglia, Erickson, Spitler, \& Amaral, 2007). This structure, which has been implicated in the detection of external threats (e.g. snakes) (Öhman, 2005) and other ecologically relevant stimuli categories (Sander, Grafman, \& Zalla, 2003), 
receives direct input from temporal visual areas (Rolls, 2007; Stefanacci, \& Amaral, 2002; Lori, Akbudak, Shimony, Cull, Snyder, Guillory, \& Conturo, 2002) such as the fusiform gyrus (e.g. Faivre, et al., 2012), which in turn receives significant input from occipital visual areas (McDonald, 1998), biased for central vision (Strasburger, Rentscheler, \& Jüttner, 2011; Kanwisher, 2001). In any case, the role of the amydgala in processing social aspects of emotion such as in recognition of facial expressions is undisputed (Buchanan, et al., 2009; Whalen, et al., 2009). In line with this view both invasive and non-invasive studies have previously shown that it responds strongly to human and even animal faces (Mormann, Dubois, Kornblith, Milosavljevic, Cerf, Ison, et al., 2011; Blonder, Smith, Davis, Kesler-West, Garrity, Avison, et al., 2004).

Unsurprisingly, most studies of emotional processing have used central presentation of faces (e.g. Heutink, Brouwer, de Jong, \& Bouma, 2011; Padmala, Lim, \& Pessoa, 2010; Vuilleumier, Armony, Driver, \& Dolan, 2003; Whalen, Shin, McInerney, Fischer, Wright, \& Rauch, 2001; Morris, Öhman, \& Dolan, 1999). However, relevant stimuli that require a rapid response also arise from the visual periphery (e.g. snakes, threatening animals) (e.g. Thorpe, Gegenfurtner, Fabre-Thorpe, \& Bülthoff, 2001). In this case, visual input is limited by receptive field properties in the peripheral retina, which are fast but convey mainly coarse information, from the magnocellular pathway (Dacey, \& Petersen, 1992). Crowding effects and reduced acuity thus weakens the discrimination of fine-grained details (e.g. facial expressions of fear and surprise expressions share many features) (Strasburger, Rentscheler, \& Jüttner, 2011). Peripheral processing often requires the superior colliculus (SC) and the pulvinar - two structures thought to be involved in a subcortical pathway to the amygdala for fast and often implicit emotional processing (Morris, et al., 1999; Vuilleumier, et al., 2003), although this is still debated in 
humans (for recent evidence in favor of this view see Tamietto, Pullens, de Gelder, Weiskrantz \& Goebel, 2012). Due to its role in threat detection, connections with SC and sensitivity to coarse information, it has been suggested that the amygdala might have a bias for peripheral faces (Bayle, Henaff, \& Krolak-Salmon, 2009; Preibisch, Lanfermann, Wallenhorst, Walter, \& Erk, 2009; Palermo, \& Rhodes, 2007). In a MEG study, early onset amygdala responses to fearful faces have accordingly been found preferentially at peripheral locations compared to central ones (Bayle, et al., 2009). However, such peripheral preference was not found in recent fMRI work (Morawetz, et al., 2010, 2011), and is not consistent with the known major connections with central vision input regions described above (e.g. the fusiform gyrus).

Only a few studies have addressed the neural correlates of central and peripheral processing of facial expressions (Preibisch, et al., 2009; Bayle, et al., 2009). The pattern of results suggested a complex interaction between facial expression type and spatial location across multiple brain regions (e.g. Preibisch, et al., 2009). A magnetoencephalography (MEG) study performed by Liu and Ioannides (2010) found faster peripheral responses but stronger central amplitudes, which is slightly at odds with the study of Bayle and colleagues (2009). Some of these inconsistencies might be related to differences between tasks. Preibich et al. (2009) required passive viewing of the emotional faces only, whereas Bayle et al. (2009) masked fearful face stimuli and asked the participants to detect happy faces. Morawetz et al. (2010) manipulated both attentional load (high, low) and task type (implicit or explicit emotion). Finally, Liu and Ioannides (2010) explicitly required participants to verbally name the emotion displayed.

Studies suggesting the existence of specific pathways for implicit and explicit emotional processing have also generated another longstanding debate, with no consensus if the amygdala 
is preferentially involved when implicit processing of threat is required, or when this emotional information is the focus of attention (explicit processing). Some studies have suggested that explicit labelling recruits cortical temporal and frontal regions thus inhibiting activity of subcortical structures such as the amygdala, which are more prone to respond when the task requires only matching of faces (Hariri, Bookheimer, \& Mazziotta, 2000), gender classifications (Critchley, et al., 2000) or passive viewing (Morawetz, et al., 2010; Taylor, Phan, Decker, \& Liberzon, 2003; for a review see Costafreda, Brammer, David, \& Fu, 2008). Nevertheless, some studies have found significant engagement of the amygdala during both during implicit and explicit tasks (Winston, O’Doherty, \& Dolan, 2003), or even enhanced activity of the left (Gorno-Tempini, Pradelli, Serafini, Pagnoni, Baraldi, Porro, et al., 2001) or bilateral amygdala (Habel, Windischberger, Derntl, Robinson, Kryspin-Exner, Gur, et al., 2007; for a review see Fusar-Poli, Placentino, Carletti, Landi, Allen, Surguladze, et al., 2009) when explicit emotional processing is required.

Subcortical structures beyond the amygdala such as the basal ganglia have not been as widely studied as the amygdala in terms of its role in visual processing of affective information. However, they have been implicated in affective processing (Arsalidou, Duerden \& Taylor, 2012), namely but not exclusively in the processing of disgust in faces (Sprengelmeyer, Young, Sprengelmeyer, Calder, Rowland, Perrett, et al., 1997). These regions connect with the amygdala in both monkeys (Fudge, Kunishio, Walsh, Richard, \& Haber, 2002) and humans (Kim, \& Whalen, 2009), and show parallel activations with the amygdala in studies of human reward and goal-oriented behaviour (O’Doherty, 2004). Our recent study in a clinical model of basal ganglia dysfunction also suggests a contribution of the basal ganglia in general face emotion recognition (van Asselen, Júlio, Januário, Campos, Almeida, Cavaco, et al., 2012). 
Concerning explicit vs. implicit processing activity within the basal ganglia, it seems to be modulated by task, with the left putamen showing stronger responses to fearful than to neutral faces during passive viewing, but to neutral than to fearful during explicit emotion judgments (Lange, Williams, Young, Bullmore, Brammer, Williams, et al., 2003), although another study suggested its involvement both during explicit or implicit discrimination of angry and happy faces (Critchley, et al., 2000). In addition, the right neostriatum (putamen and caudate) was activated when subjects made explicit judgements of disgust, with the right caudate (head) differentiating between disgusted and happy faces (Gorno-Tempini, et al., 2001) or being generally involved in explicit judgements (Fusar-Poli, et al., 2009).

In sum, the role of amygdala in emotion processing does remain controversial (for reviews see Öhman, 2009; Pessoa \& Adolphs, 2010; Tamietto \& de Gelder, 2010) and the link with basal ganglia function remains also intriguing. Here we studied animal face recognition and threat detection using stimuli presented either at foveal regions or at near-periphery locations $\left(<10^{\circ}\right)$, although we will refer here to the near-periphery as peripheral vision (see also Strasburger, et al., 2011).

The main goals of this study were to investigate the neural correlates underlying central and peripheral processing of threat relevant stimuli, and in particular test the peripheral bias hypothesis with stimuli that are ecologically relevant for human emotional cognition (animal faces). We hypothesize that different regions may be recruited for central and peripheral processing of faces, given the likely reorganization of amygdala input from foveally biased areas. Since in primates, faces are preferentially processed in the fovea, we also hypothesize amygdala preference for faces presented at central locations. Additionally, we aimed to study the dissociation between automatic/implicit vs. more controlled/explicit processing of threat relevant 
information and the role of the amygdala and other regions, such as the basal ganglia, in those processes. 


\section{Materials and methods}

\subsection{Participants}

Twenty healthy participants (age range 19-34, mean [SD] age 26.30 [4.54], 10 males) took part in the study. All subjects were right handed except 1 (ambidextrous) and all had normal or corrected-to-normal vision. All gave written informed consent, according to the Declaration of Helsinki, and the experimental protocol was approved by the ethics committee of the Faculty of Medicine of the University of Coimbra.

\subsection{Stimuli and apparatus}

Pictures of animal faces and natural displays were used as stimuli. Two types of animal faces were used: threatening animal faces (e.g. wolves, bears, dogs, sharks, tigers, leopards) displaying the mouth open and showing their teeth; and non-threatening animal faces (e.g. horses, sheep, rabbits, cows), displaying a neutral facial expression and mouth closed. A third set of stimuli, control non-faces, displaying non-facial natural scenes (e.g. grass, plains, meadows, flowers), was used as a baseline size matched control set. The images were taken both from the internet and the International Affective Picture System (IAPS) set (CSEA-NIMH, USA, csea.phhp.ufl.edu), and were manipulated in such a way that the animal face was centred in the picture display. Each picture was presented within a squared shape, yielding a visual angle of $6.84^{\circ} \times 6.84^{\circ}(\mathrm{W} \times \mathrm{H})$, and presented at one of three possible locations: centre, $0^{\circ}$, right or left, $7.71^{\circ}$. A prior validation study was performed for stimulus selection. A total of 110 pictures (55 containing animal faces, 55 containing control non-face stimuli) were presented at peripheral locations (both right and left) during $150 \mathrm{msec}$. Twelve participants responded if they could 
recognize an animal in the picture, and were requested to rate the pictures in terms of valence (positive, negative or neutral) / arousal (5-point scale) ratings. A final set of 48 pictures were selected. Threatening faces were rated as negative (valence mean $[\mathrm{SD}]=-0.49[0.42]$, range: -1 to 1 ) and with mean [SD] arousal ratings of 1.57 [0.38], range: 0 to 5), while the non-threatening animal faces were rated as positive (valence mean $[\mathrm{SD}]=0.68[0.23]$, range: -1 to 1 ), and having a mean [SD] arousal rate of 1.38 [0.56], range: 0 to 5). Twenty four baseline size matched control stimuli were also used. Inside the scanner, the stimuli were back projected using an AVOTEC (www.avotec.org) projector on a 20(w) x 15(h) (1024 x 768 pixels) screen pad that was placed at a viewing distance of $50.5 \mathrm{~cm}$ by means of a head coil mounted mirror. The tasks were presented using Presentation software (Neurobehavioral Systems, USA, www.neurobs.com), and originally displayed on a monitor with a $60 \mathrm{~Hz}$ refresh rate. Responses were given by means of a response box (Cedrus Lumina LP-400 response pad for fMRI, www.cedrus.com).

\subsection{Task design and procedure}

An fMRI slow event-related design was performed with 4 sequential runs of 54 trials each $(4 \mathrm{x}$ 217 volumes $)$. Each trial started with a fixation cross $(500 \mathrm{msec})$ followed by a picture $(150$ msec) presented in central, left or right locations of the screen. The participants had to press one of two buttons, according to the task to perform. An inter-trial interval (ITI) matched with the Repetition Time (RT, $2500 \mathrm{msec}$ ) followed the picture presentation and varied randomly (7.5, $10,12.5 \mathrm{sec}$ ) (see Figure 1). Participants were asked to remain as still as possible during the testing session. It was emphasized that this would be important in order to minimize data artefacts. Importantly, different tasks were performed while fixating a central cross: (a) to report 
presence of an animal face (task 1: 'implicit threat' animal face recognition, first 2 runs) or (b) to detect threat signals (task 2: 'explicit threat' detection, last 2 runs) by means of a 2-button (Yes/No) response box. Picture duration was kept short to prevent visual saccades and eye movements were recorded (MR compatible AVOTEC/SMI systems) to ensure central fixation.

\subsection{Imaging data acquisition and preprocessing}

Functional images were acquired in a 3T Siemens TimTrio scanner using BOLD contrast echo planar imaging (EPI, TR=2.5, TE=49, 29 4mm-thick-slices with no inter-slice gap, with an inplane matrix of $128 \times 128$ voxels) covering the entire brain. The scanning session also included a high resolution T1 weighted anatomical scan (MPRAGE sequence, 1x1x1 mm3 voxel size, TR $2.3 \mathrm{~s}$, TE $2.98 \mathrm{~ms}, 160$ slices) to help in the transformation of the functional images into standard space. The data were preprocessed and analyzed using BrainVoyager QX v2.4 32-bit (Brain Innovation, www.brainvoyager.com). Preprocessing included slice scan time corrections, temporal filtering and motion correction. Before group analysis the images were spatially smoothed using a 4-mm full-width-half-maximum Gaussian kernel and then transformed into Talairach space.

\subsection{Statistical analyses}

All the statistical analyzes were performed using IBM SPSS Statistics 19 and 20 (IBM, USA, http://www.ibm.com/software/analytics/spss/) and the Brain Voyager v2.6 software. The computation of effect sizes and power was performed with $\mathrm{G}^{*}$ Power 3.1.6 (Faul, Erdfelder, Lang, \& Buchner, 2007). 


\subsubsection{Behavioural data}

Data from behavioural reports were considered to classify the trials where correct responses occurred. Therefore, trials corresponding to misses and false alarms (e.g. trials with: no response, threatening and non-threatening faces not recognized, or non-threatening faces and natural displays categorized as threatening) were excluded from the present analysis, but included in the design model of the functional data analysis as confound predictors. Accuracy measures, observer's $d$ prime measures ( $d^{\prime}$ ) and reaction times (RTs) were obtained. The Accuracy was computed in order to have a measure of correct performance, whereas the $d$ prime measure, which assesses response sensitivity, was computed in order to control for subject response bias (Stanislaw, \& Todorov, 1999; Provost, \& Fawcett, 1997). Both the Accuracy and the index $d$ ' measures were computed for each task and spatial location. For the Accuracy measure we used hits, false alarms, misses and correct rejections in the following formula: Accuracy $=[$ hits + correct rejects] / [hits + false alarms + misses + correct rejects]. For the index $d$ ' we used the subsequent formula: Z(hits) - Z(false alarms), using the idf.norm function of the IBM SPSS software. For the RT measure, we compared between tasks ('implicit threat' animal face recognition, 'explicit threat' detection), spatial locations (centre, left, right) and stimulus types (threatening animal face, non-threatening animal face, control non-face). One participant was excluded from the behavioural analysis due to timing issues in response time collection. Due to the non-normal distribution of data, non-parametric tests were used in all the analyses (Friedman and Wilcoxon signed rank tests for related samples)

\subsubsection{Functional data}


Statistical analyses were performed using a random effects general linear model (GLM) approach. Event duration was set to $4 \mathrm{sec}$ beginning in the stimulus onset. Both spatial location (centre, left, right) and stimulus type (threatening animal faces, non-threatening animal faces, and control non-faces) were manipulated, with 9 predictors being included in each single-subject's design matrix (spatial location $\mathrm{x}$ stimulus type). A box car function was defined for each predictor and convolved with a canonical hemodynamic response function.

Two different analyses were then carried: region of interest and whole brain analyses. First, two regions of interest (ROIs) were defined in the left and right amygdalae of each participant based on anatomical landmarks (Duvernoy, 1999) (see Supplementary Figure A.1). Parameter estimates (z-normalized beta weights) were computed for each ROI and each task, with ANOVAs random effects (RFX) and post-hoc $t$-tests being performed using the IBM SPSS software. When applicable, corrections of Greenhouse-Geisser were reported together with tests of sphericity. Planned RFX-GLM contrasts analyses were performed using BrainVoyager.

Second, whole brain analyzes were performed for each task separately and for direct comparison of both tasks. The statistical maps display specific contrasts after a whole brain RFX-GLM analysis being computed with brain mask restriction (53842 voxels). Corrections for multiple comparisons were made through the Cluster Threshold plugin (BrainVoyager) using 1000 Monte Carlo simulations. Minimum cluster sizes corresponding to significance at a threshold of $p<.01$ were computed for each contrast.

\section{Results}

\subsection{Behavioural data}


The participants performed two different tasks. In the first task ( 'implicit threat' animal face recognition task), they were asked to report (yes/no) if the picture presented contained an animal face, while in the second task ('explicit threat' detection task) they were required to report an yes/no answer regarding the detection of threat signals in the picture. For the Accuracy and Sensitivity index ( $\left.d^{\prime}\right)$ analyses (see Methods), we compared performance between tasks ('implicit threat' animal face recognition or 'explicit threat' detection) and spatial locations (centre, right, and left). For the reaction time analysis, we further included stimulus type (threatening animal face, threatening animal face, and control non-face) as a factor.

\subsubsection{Accuracy}

Accuracy across participants was above 98\%, for task 1 ('implicit threat' animal face recognition task), and above $97 \%$ for task 2 ('explicit threat' detection task), with differences in Accuracy not reaching significance (Wilcoxon paired test, $\mathrm{W}=90.500, \mathrm{Z}=-.182$, n.s.; 2-tailed).

The participants were able to recognize an animal face (task 1) presented in the centre (mean $[\mathrm{SD}]=.98[.03]$ ) or in peripheral locations (left: mean $[\mathrm{SD}]=.98$ [.02]; right: mean $[S D]=.97[.04])$ with a high level of accuracy. Likewise, they were able to accurately detect threat in threatening animal faces (task 2) independently of location of presentation (centre: mean $[\mathrm{SD}]=.97[.04]$; left: mean $[\mathrm{SD}]=.97$ [.04]; right: mean $[\mathrm{SD}]=.97$ [.04]). Friedman tests performed separately for each task showed that were no differences for spatial location when discriminating between stimulus type $\left(\operatorname{task} 1: \chi^{2} \mathrm{~F}(2)=1.192\right.$, n.s.; $\operatorname{task} 2: \chi^{2} \mathrm{~F}(2)=2.333$, n.s. $)$. To see if a difference between tasks occurred as a function of spatial location, we performed Wilcoxon paired tests between task 1 and task 2 at each location. These turned out non- 
significant for any of the spatial locations (centre: $\mathrm{W}=58.500, \mathrm{Z}=-.495$, n.s.; left: $\mathrm{W}=53.500, \mathrm{Z}=-$ .759 , n.s.; right: $\mathrm{W}=53.000, \mathrm{Z}=.032$, n.s.; 2-tailed tests).

In this manner, we can conclude the participants responded to the pictures as expected, and no dissimilar performances neither across tasks nor spatial locations were found.

\subsubsection{Sensitivity index (d')}

To further assess accuracy of performance was not affected by response bias, we have further tested "corrected" accuracy across tasks by using the bias free classical $d$ prime measure. This measure computes the observer's sensitivity to detect a signal taking into consideration the false alarms rate when controlling for bias (e.g. animal face, threat).

The results indicated no differences in $d$ ' measures between task 1 and task 2 (task $1>$ task 2: Wilcoxon paired test, $\mathrm{W}=126.000, \mathrm{Z}=.784$, n.s., 2-tailed). Again, Friedman tests performed separately for each task displayed no differences for spatial location $\left(\operatorname{task} 1: \chi^{2} \mathrm{~F}(2)=\right.$ .724, n.s.; task2: $\chi^{2} \mathrm{~F}(2)=4.651$, n.s. $)$. Additionally, Wilcoxon paired tests showed no differences between tasks at each location (centre: $\mathrm{W}=54.500, \mathrm{Z}=-.698$, n.s., 2-tailed; left: $\mathrm{W}=46.000, \mathrm{Z}=-$ 1.140, n.s., 2-tailed; right: $\mathrm{W}=64.000, \mathrm{Z}=.227$, n.s.; 2-tailed tests).

Therefore, the accuracy data is not influenced by response bias, given the results from the sensitivity index $d$ '. We can conclude that the performance was globally matched in what concerns task type and spatial location.

\subsubsection{Reaction time (RT)}

For the RT measure, Friedman tests showed neither differences between tasks (mean [SD] RT task $1=804.40$ [132.44] msec; mean [SD] RT task $2=882.50$ [179.99] msec; $\mathrm{W}=143.000$, 
$\mathrm{Z}=1.932, p=.053$; although a trend was found for higher RT during task 2) nor an effect of spatial location (task $1: \chi^{2} \mathrm{~F}(2)=2.842$, n.s.; task $2: \chi^{2} \mathrm{~F}(2)=2.632$, n.s. $)$. However, a main effect of stimulus type was found in both tasks $\left(\operatorname{task} 1: \chi^{2} \mathrm{~F}(2)=7.895, p=.019 ; \operatorname{task} 2: \chi^{2} \mathrm{~F}(2)=7.053\right.$, $p=.029)$. Post-hoc paired sample test revealed differences in the contrasts 'non-threatening faces $>$ threatening face' $(\mathrm{W}=-.789, \mathrm{Z}=-2.433, p=.045$, Cliff's delta $=.197$; corrected for multiple comparisons) and 'non-threatening face $>$ control non-faces' ( $\mathrm{W}=.789, \mathrm{Z}=2.433, p=.045$, Cliff's delta $=.197$; corrected for multiple comparisons) during the 'implicit threat' animal face recognition task, and for the contrast 'threatening faces $>$ control non-faces' ( $W=.842, \mathrm{Z}=2.596$, $p=.028$, Cliff's delta $=.263$; corrected for multiple comparisons) during the 'explicit threat' detection task.

\subsection{Functional MRI data}

Region of interest (amygdala) and whole brain random effects general linear model (RFX-GLM) analyses were performed.

\subsubsection{Region of interest (ROI) analysis: The amygdala}

We performed 3x3 ANOVAs RFX for each task ('implicit threat' animal face recognition or 'explicit threat' detection) in each amygdala ROI. Spatial location (centre, right, and left) and stimulus type (threatening animal face, threatening animal face, and control non-face) were taken as factors (Figures 2, 3 and 4). 


\subsubsection{Main effects}

For the 'explicit threat' detection task (task 2), a main effect of spatial location was found for the right amygdala $(\mathrm{F}(2,38)=3.533, p=.039$, Cohen's $d=.432$, power $(1-\beta)=.999)$. During the 'implicit threat' animal face recognition task (task 1), a main effect of stimulus type was found for the left amygdala $(\mathrm{F}(2,38)=4.103, p=.024$, Cohen's $d=.465$, power $(1-\beta)=1.000)$, as well as a marginal effect of spatial location $(\mathrm{F}(2,38)=3.194, p=.052$, Cohen's $d=.410$, power $(1-\beta)=.999)$.

Posthoc analyses showed the differences in the right amygdala ROI emerged from the contrast 'central > left' $(t(19)=2.733, p=.013$, Cohen's $d=.611$, power(1- $\beta)=.839)$, the same contrast was also underlying the marginal effect of spatial location in the left amygdala during task $1(t(19)=2.694, p=.042$, corrected for multiple comparisons, Cohen's $d=.552$, power $(1-$ $\beta)=.769$ ). Regarding the effect of stimulus type, the left amygdala differences were found for the contrast 'threatening animal faces > control non-face: $t(19)=2.375, p=0.028$, Cohen's $d=.465$, power $(1-\beta)=.999)$.

\subsubsection{Interaction effects}

An interaction effect between spatial location and stimulus type was found in the right amygdala $(\mathrm{F}(2,850 ; 54,144)=3.180, p=0.033$, Cohen's $d=.409$, power $(1-\beta)=.999$, Greenhouse-Geisser correction, Mauchly's W(9)=.263, $p=.006, \varepsilon=.712$ ) for the 'explicit threat' detection task.

Posthoc tests revealed differences between animal faces and control non-faces only for centrally presented stimuli (centre: threatening animal faces $>$ control non-faces: $t(19)=3.701$, $p=0.001517$, Cohen's $d=.828$, power $(1-\beta)=.973$; non-threatening animal faces $>$ control nonfaces: $t(19)=3.341, p=0.003432$, Cohen's $d=.747$, power $(1-\beta)=.942)$. 


\subsubsection{Whole brain RFX analysis}

We performed whole brain RFX contrast analyses to identify brain regions involved in task and spatial location effects (brain regions, peak voxel coordinates and statistics are presented in Table 1).

\subsubsection{Task: 'implicit threat' animal face recognition vs. 'explicit threat' detection}

Differences among tasks become apparent in the right fusiform gyrus, right cuneus, left lingual gyrus, left medial frontal gyrus, left putamen, left middle temporal gyrus and left cerebellum, with increased activity during the 'explicit threat' detection task for these regions (see Figure 5 and Table 1 - contrast a).

\subsubsection{Spatial location: centre vs. periphery}

Whole brain RFX planned contrasts performed for the effect of spatial location revealed increased activity in the right fusiform gyrus, left superior frontal gyrus and left middle temporal gyrus in central compared to peripheral stimulation. In the contrary, the right posterior cingulate gyrus responded more to peripherally presented stimuli (see Table 1 - contrast b).

\subsubsection{Task $x$ Spatial location}

We identified a surprising difference in striatal activation patterns across tasks. We directly compared performance accross spatial locations between task 1 and task $2(n=20)$.

Central representations: concerning areas that activate more strongly for task 2 (threat detection) we observed that when stimuli were presented centrally (Figure 6, top), significant 
differences were in seen the right caudate head of the basal ganglia and in the left lingual gyrus, (Table 1 - contrast c)

Peripheral representations: for peripheral presentations (Figure 6, bottom), between task differences sowed stronger activity during task 2 in the left putamen, right fusiform gyrus, right posterior cingulate and bilateral cerebellum (Table 1 - contrast d).

The data shows a task dependent centre vs. periphery bias in visual and importantly, also in striatal regions. Given the pattern found in the basal ganglia, planned post-hoc tests were then performed. Therefore, first we contrasted only the task-relevant conditions. The contrast 'threatening animal faces (task 2)' > 'animal faces (threatening + non-threatening) (task 1)' for central presentations yielded differences in the right caudate $(t(19)=4.521, p=0.000234 ; \mathrm{x}=11$, $\mathrm{y}=7, \mathrm{z}=3)$ and in the right $(t(19)=4.993, p=0.000081 ; \mathrm{x}=18, \mathrm{y}=-4, \mathrm{z}=7)$ and left putamen $(t(19)=4.260, p=0.000423 ; \mathrm{x}=-24, \mathrm{y}=-1, \mathrm{z}=10)$, matching the original pattern of results. For peripheral stimuli the contrast 'threatening faces' (task 2) $>$ 'animal faces (threatening + nonthreatening)' (task 1) returned a difference only in the left putamen $(t(19)=3.638, p=0.001749$; $\mathrm{x}=-16, \mathrm{y}=10, \mathrm{z}=6$ ). Most important is that the contrast 'non-threatening (face + non-face)' (task 2) > control non-face' (task 1) did not yield any significant pattern, suggesting that the basal ganglia findings are specific for task-relevant stimuli. 


\section{Discussion}

The main goals of this work were twofold: first, to investigate the hypothesis of a potential central bias in the amygdala for processing of facial stimuli, given that its major input comes from foveally-biased ventral visual areas. Second, we studied a possible dissociation in the neural correlates of central and peripheral threat processing, and how task instructions can modulate information processing. Although we focused on the role of amygdala as a region of interest, we also performed whole-brain analyses to understand face recognition and threat processing at a more general level.

We identified both in ROI and whole brain analysis task related activity differences (animal face recognition vs. threat detection) on a spatial location (central vs. peripheral emotional) dependent basis. Accordingly, we have found evidence for distinct regions involving explicit vs. implicit processing, with an emphasis on the amygdala and the striatum. In the amygdala, no response bias to peripheral locations was found using face stimuli. Moreover we found that these responses were task (implicit vs. explicit) and hemisphere dependent. In the basal ganglia structures we also identified strong task and location dependence. Indeed, one of the most important findings of this work was that central and peripheral 'threat vs. animal face' task differences recruited different basal ganglia regions: central information involved the caudate head and peripheral information engaged mainly the putamen. These findings are interesting in the way they may contribute for the scarce but stimulating body of evidence that implicates basal ganglia in affective aspects of visual processing.

This study bears implications on the understanding of implicit vs. explicit processing of emotional information as a function of spatial position (central or peripheral). 


\subsection{The amygdala}

\subsubsection{A central bias for faces in the amygdala}

Different object categories have specific eccentricity biases, with face stimuli being preferentially processed within central vision (Levy, et al., 2001). The amygdala receives major input from foveally-biased ventral areas. For this reason, we hypothesized that it might show increased activity for face-objects presented at foveal locations, in spite of the conventional view (Palermo \& Rhodes, 2007). Our results are in line with our prediction, with central compared to left visual field face presentations eliciting stronger activation in particular in the right amygdala during the explicit threat task.

Some studies (Bayle, et al., 2009; Preibisch, et al., 2009; Palermo \& Rhodes, 2007) have nevertheless suggested that, due to its potential connections with the SC and the pulvinar, which are more related with magnocellular pathways and processing of low spatial frequency information, a bias might arise for peripheral processing of negative (e.g. fear) facial expressions. It must be pointed however that while the ratio parvocellular/magnocellular projections is high for stimuli processed in the fovea, in the periphery both magnocellular and parvocellular processing are significant (Azzopardi, Jones \& Cowey, 1999). In any case, in our study no peripheral bias was found. This is at least partially consistent with previous results using fearful faces (Morawetz, et al., 2011), which found a lack of modulation concerning spatial location, and in substantial agreement with another study (Morawetz, et al., 2010) which found a difference central $>$ periphery during the performance of relatively low attentional load tasks, regardless of whether they were implicit (matching digits) or explicit (matching emotion). 
Two factors should be discussed here. First, different eccentricities were used across studies, with the difference between central and peripheral locations arising at $5.6^{\circ}$ of visual angle, but not at $11.25^{\circ}$ (Morawetz, et al., 2010) nor at 9.5 (Morawetz, et al., 2011). In our study, we used an intermediate visual angle, $7.71^{\circ}$, which is more close to the study reporting a central bias. Second, magnification factors (used in Morawetz, et al., 2011, 2010; Liu \& Ioannides, 2010; Preibisch, et al., 2009 but see Bayle, et al., 2009) may be an issue. We addressed this issue by using control non-face stimuli that were scale matched to the face stimuli. The former did not show the central bias found with faces. This approach showed that stimulus type was more relevant than scaling in explaining our pattern of results. Moreover, task and hemispheric dependencies are not easily explained by magnification differences. Previous studies confirmed the possibility to study early amygdala activity at peripheral locations even when no scaling is used (Bayle, et al., 2009), an approach that we also followed (for a review on the role of low level properties see Strasburger, et al., 2011).

The central face bias in the amygdala might be explained by centrally-biased inputs from areas along the occipital-temporal cortex belonging to the face network (e.g. Rolls, 2007). Accordingly, our whole brain data showed increased activity in the right lateral occipital (LO) during central presentations. Moreover, the contrast of explicit over implicit threat seemed to engage more strongly the right occipital gyrus. Together, these findings provide some insight to understand why the right amygdala showed a specific response at central spatial locations to both threatening and non-threatening faces in particular during the explicit threat task. In contrast, the left amygdala, although showing a difference between threatening animal faces from non-faces, this happened irrespective of spatial location (accordingly, no left occipito-temporal areas 
showed a spatial location bias). These observations support the notion major involvement of right hemispheric specialized areas in foveal face processing (Kanwisher, et al., 1997).

\subsubsection{The amygdala responds to the threatening content of animal faces}

We found an overall increased response of the left amygdala to threatening animal faces compared to control non-faces, irrespective of spatial location, during the 'implicit threat' animal face recognition task, whereas during the 'explicit threat' detection task, the right amygdala differentiated between faces and non-faces only when centrally presented.

In our study we made use of threatening animal faces whereas most of the previous studies have used fearful human faces. Two points should be addressed, in this context. First, some studies have suggested that the amygdala responds differently to fearful and angry emotional faces. Overall angry faces might elicit stronger responses in particular amygdala nuclei (such as the corticomedial), being positively correlated with increased behavioural reports of perceived threat (Boll, Gamer, Kalisch, \& Büchel, 2011; but see opposite findings in Whalen, et al., 2001). As a second point, threatening signals in animal and human faces are most likely species-specific (e.g. Gothard, et al., 2007). Therefore, direct comparisons between previous studies with human faces may not be feasible. In fact, we decided to use animal instead of human faces because both have distinct ecological value and since direct recordings suggest the amygdala responds surprisingly stronger to animal faces than to human (irrespective of facial expression) faces (Mormann, et al., 2011), as they might have an increased survival value.

It has been shown that activity within the amygdala declines with repeated presentations, an effect attributed to stimulus familiarity (Wilson, \& Rolls, 1993). However, we have reasons to believe this was not an issue in our study because stimuli were not repeated. It 
might also be pointed that the amygdala shows a preferential response to the 'threatening' animals simply because these act as 'new' stimuli, in comparison with non-threatening stimuli. This is unlikely given the nature of our sampling strategy. Moreover our data shows that this region of the brain responds to both animal categories, in agreement with previous results (Mormann, et al., 2011).

\subsubsection{The amygdala shows a lateralized response depending on task instructions}

Differences regarding task related activity were related to a lateralized central vs. peripheral amygdala preference for threatening faces. In fact, our results point to a central preference in the right amygdala during the explicit threat task and to a left lateralized amygdala response during the implicit threat (animal face recognition) task. This might at first glance seem counterintuitive, as right amygdala has been originally more related with automatic and implicit processing (e.g. Morris et al., 1999), unlike the left amygdala (Gorno-Tempini, et al., 2001). However these views can be reconciled if one considers that our study took into account how central vs. peripheral responses in the amygdala may interact with task instructions.

The controversy regarding the role of the amygdala in implicit processing is well recognized (e.g. Hariri, et al., 2000). Other authors (Öhman, 2009; Tamietto \& de Gelder, 2010) have hypothesized a prominent role of the amygdala in automatic emotional processing, in relation to coarse recognition of relevant information routed through the pulvinar and the superior colliculus (Tamietto, et al., 2012). It has been recognized that task demands might modulate the amygdala response (Pessoa, McKenna, Gutierrez, \& Ungerleider, 2002; Costafreda, et al., 2008), in addition to the fact that the pulvinar has also been related to conscious attentional processes (Padmala, et al., 2010). Our work provides a new perspective on 
this view by showing that the amygdala may also show task dependent responses to both explicit and centrally processed stimuli. Our findings are therefore in agreement with a recent review which has pointed to major involvement of the amygdala in explicit processes (Fusar-Poli, et al., 2009).

In any case, our findings add to the ongoing discussion on the left/right amygdala lateralization patterns. It has been proposed that the right amygdala responds when the emotional property of the stimulus is visual and directly obvious to the subject, while the left would show preference for verbally learned stimuli (Phelps, et al., 2001). Also, the intriguing study of Heutink, Brouwer, de Jong \& Bouma (2011) have found that absence of the right amygdala impairs the overt, explicit, identification of fear, while covert, implicit, recognition of fear and aversive conditioning may still occur following lesion of the right amygdala. Notably, stimulus type (face vs. control non-faces) and task seem to influence patterns of activity and lateralization in the amygdala (but see Baas, Aleman, \& Kahn, 2004).

\subsection{The basal ganglia}

To our knowledge there are very few studies addressing directly the relation between the amydgala, basal ganglia and central vs. peripheral emotion processing. Morawetz and colleagues (2010) addressed the question of how spatial location, and attentional load modulate particular brain regions by using a ROI-based approach centred only in the amygdala and the fusiform gyrus, which did not allow them to explore the functional role of other regions.

\subsubsection{Different neural correlates for central and peripheral visual emotion recognition}


In our study, we presented angry and neutral animal facial expressions both in the centre and in visual periphery. We found that peripheral and central processing of visual threat signals do correspond to different brain networks. Our results showed that peripheral processing recruited mainly the putamen, which is known to be dominantly related to implicit processing (Rauch, Whalen, Savage, Curran, Kendrick, Brown, et al., 1997), whereas the caudate was only involved during central stimuli appraisal. This region is relatively more involved in explicit goal oriented processing (Brown, Redondo-Verge, Chacon, Lucas, \& Channon, 2001; Ruge \& Wolfensteller, 2010).

One MEG study, with inherent limitations in the interpretation of activity in deep structures and their subparts, has partly addressed this issue by suggesting an involvement of the thalamus, amygdala and basal ganglia in the rapid detection of threat (Luo, Holroyd, Jones, Hendler, \& Blair, 2007). However, this pattern was found for fearful but not for angry or neutral expressions. Furthermore, faces were only presented centrally. To our knowledge, only one study showed striatum activity with peripheral presentation of static (happy $>$ neutral) faces (Faivre, et $a l .$, 2012). However, several methodological differences with our study were present, as the caudate was specifically found in our study to be modulated by the difference between explicit threat detection and simple animal face recognition tasks, whereas Faivre and colleagues (2012) studied only implicit processing of happy vs. neutral faces.

Importantly, the caudate head seems to receive and project for several areas along the visual cortex, in particular inferotemporal (Baizer, Desimone, \& Ungerleider, 1993; Saint-Cyr, Ungerleider, \& Desimone, 1990). This might in part explain why it plays a major role at central spatial locations. Different functions have been attributed to the caudate head and the putamen, with the caudate more engaged in emotional (Arsalidou, et al., 2012) and goal-oriented 
processes, whereas the putamen appears to subserve more automatic cognitive functions (Grahn, Parkinson, \& Owen, 2008).

\subsubsection{An explicit > implicit bias goal-oriented response in the basal ganglia}

Our findings are in agreement with reports of increased right caudate activity for explicit compared with implicit emotional processing (for a review, see Fusar-Poli, et al., 2009). In fact, in our study the caudate part of the striatum was consistently found to respond more to threatening animal faces during the explicit task than to neutral (non-threatening) animal faces (bilateral caudate) or non-facial displays (right caudate), which is consistent with its role in conscious emotional processes. The fact that the putamen activated more strongly for the explicit threat task might however challenge its preferential involvement in implicit processes (at least when spatial central-periphery constraints are not taken into account). Nevertheless, other authors have found increased left putamen for explicit emotional tasks (Critchley, et al., 2000; Sugiura, et al., 2000), with bilateral putamen responding to implicit tasks (Critchley, et al., 2000). It is possible that the right and the left putamen play different roles in emotional processing. Here we found an interaction of basal ganglia structures with spatial location, with the left putamen activating preferentially to explicit threat mainly in the periphery.

\subsection{Limitations}

Potential differences between our study and others (Liu \& Ioannides, 2010; Bayle, et al., 2009) might arise from the methodologies used. In fact, the temporal resolution of MEG is much higher than the one currently used in our fMRI study, although the latter has better spatial resolution. The former point is nevertheless an important point, as the lack of amygdala responses for 
peripheral stimuli as measured in fMRI does not mean that this type of processing does not occur. In fact, one might argue about detection sensitivity: the peripheral response might occur earlier and faster, and/or with diminished amplitude as compared to more central and explicit processing. Moreover, the differences in the left amygdala for responses to threatening vs. nonface stimuli might suggest an automatic role of the amygdala, adding to the evidence for a role on conscious emotional processing.

Although different amygdala subnuclei were proposed to be involved in the processing of angry and fearful emotional expressions (e.g. Whalen, et al., 2001), the spatial resolution (voxel dimension) chosen for our study did not allow us to individuate the contributions of each. However, recent work (e.g. Boll, et al., 2011) offers promising opportunities to study the role of different amygdala nuclei in different affective functions.

\subsection{Major conclusions}

We found a lateralized response of the amygdala as a function of task instructions, with a bias for central processing of faces occurring specifically in the (right) amygdala during explicit threat processing.

Furthermore, we found a dual striatal contribution preferentially tuned for central (caudate) or peripheral (putamen) processing of threat content information, the former being more related to goal directed processing and the later with automatic processing.

\section{Conflicts of interest}

The authors declare no conflict of interest. 


\section{Acknowledgements}

This work was supported by the following grants (Compete PTDC/PSI/67381/2006, PIC/IC/82986/2007, PEst-C/SAU/UI3282/2011, CENTRO-07-ST24-FEDER-00205, FP7HEALTH-2013-INNOVATION-1) and the BIAL Foundation. Inês Almeida was supported by an FCT grant (SFRH / BD / 35973 / 2007). The authors also want to acknowledge José Rebola and Inês Violante for support in the data analysis, and Carlos Ferreira and João Marques for technical assistance within the MR scanner.

\section{Supplementary data}

Supplementary data associated with this article can be found in the online version. 


\section{References}

Arsalidou, M., Duerden, E.G., Taylor, M.J. (2012). The centre of the brain: topographical model of motor, cognitive, affective, and somatosensory functions of the basal ganglia. Human Brain Mapping, 000, 000-000. (published ahead of print)

Atkinson, A. P., \& Adolphs, R. (2011). The neuropsychology of face perception: beyond simple dissociations and functional selectivity. Proceedings of the Royal Society of London. Series B: Biological Sciences, 366(1571), 1726-1738.

Azzopardi, P., Jones, K. E., \& Cowey, A. (1999). Uneven mapping of magnocellular and parvocellular projections from the lateral geniculate nucleus to the striate cortex in the macaque monkey. Vision Research, 39(13), 2179-2189.

Baas, D., Aleman, A., \& Kahn, R. S. (2004). Lateralization of amygdala activation: a systematic review of functional neuroimaging studies. Brain Research Reviews, 45, 96-103.

Baizer, J. S., Desimone, R., \& Ungerleider, L. G. (1993). Comparison of subcortical connections of inferior temporal and posterior parietal cortex in monkeys. Visual Neuroscience, 10(1), 59-72.

Bayle, D. J., Henaff, M. A., \& Krolak-Salmon, P. (2009). Unconsciously perceived fear in peripheral vision alerts the limbic system: a MEG study. PLoS One, 4, e8207.

Blonder, L. X., Smith, C. D., Davis, C. E., Kesler-West, M. L., Garrity, T. F., Avison, M. J., \& Andersen, A. H. (2004). Regional brain response to faces of humans and dogs. Brain Research, 20(3), 384-394.

Boll, S., Gamer, M., Kalisch, R., \& Büchel, C. (2011). Processing of facial expressions and their significance for the observer in subregions of the human amygdala. NeuroImage, 56(1), 299-306. 
Brown, R. G., Redondo-Verge, L., Chacon, J. R., Lucas, M. L., \& Channon, S. (2001).

Dissociation between intentional and incidental sequence learning in Huntington's disease. Brain, 124, 2188-2202.

Buchanan, T. W., Tranel, D., \& Adolphs, R. (2009). The human amygdala in social function. In P. W. Whalen \& L. A. Phelps (Eds.), The human amygdala (pp. 289-318). New York: The Guilford Press.

Costafreda, S. G., Brammer, M. J., David, A. S., \& Fu, C. H. Y. (2008). Predictors of amygdala activation during the processing of emotional stimuli: A meta-analysis of 385 PET and fMRI studies. Brain Research Reviews, 58, 57-70.

Critchley, H., Daly, E., Phillips, M., Brammer, M., Bullmore, E., Williams, S., Van Amelsvoort, T., Robertson, D., David, A., \& Murphy, D. (2000). Explicit and implicit neural mechanisms for processing of social information from facial expressions: A functional magnetic resonance imaging study. Human Brain Mapping, 9, 93-105.

Dacey, D. M., \& Petersen, M. R. (1992). Dendritic field size and morphology of midget and parasol ganglion cells of the human retina. Proceedings of the National Academy of Sciences of the United States of America, 89(20), 9666-9670.

Duvernoy, H. M. (Ed.) (1999). The human brain: Surface, three-dimensional sectional anatomy with MRI, and blood supply. Wien: Springer-Verlag Wien New York.

Faivre, N., S. Charron, S., Roux, P., Lehéricy, S. \& Kouider, S. (2012). Nonconscious emotional processing involves distinct neural pathways for pictures and videos. Neuropsychologia, 50(14), 3736-3744. 
Faul, F., Erdfelder, E., Lang, A.-G., \& Buchner, A. (2007). G*Power 3: A flexible statistical power analysis program for the social, behavioral, and biomedical sciences. Behavior Research Methods, 39, 175-191.

Fudge, J. L., Kunishio, K., Walsh, P., Richard, C., \& Haber, S. N. (2002). Amygdaloid projections to ventromedial striatal subterritories in the primate. Neuroscience, 110(2), $257-275$.

Fusar-Poli, P., Placentino, A., Carletti, F., Landi, P., Allen, P., Surguladze, S., Benedetti, F., Abbamonte, M., Gasparotti, R., Barale, F., Perez, J., McGuire, P., \& Politi, P. (2009). Functional atlas of emotional faces processing: a voxel-based meta-analysis of 105 functional magnetic resonance imaging studies. Journal of Psychiatry \& Neuroscience, $34,418-432$.

Gorno-Tempini, M. L., Pradelli, S., Serafini, M., Pagnoni, G., Baraldi, P., Porro, C., Nicoletti, R., Umita, C., \& Nichelli, P. (2001). Explicit and incidental facial expression processing: an fMRI study. NeuroImage, 14, 465-473.

Gothard, K. M., Battaglia, F. P., Erickson, C. A., Spitler, K. M., \& Amaral, D. G. (2007). Neural responses to facial expression and face identity in the monkey amygdala. Journal of Neurophysiology, 97(2), 1671-1683.

Grill-Spector, K., Knouf, N., \& Kanwisher, N. (2004). The fusiform face area subserves face perception, not generic within-category identification. Nature Neuroscience, 7, 555-562. Habel, U., Windischberger, C., Derntl, B., Robinson, S., Kryspin-Exner, I., Gur, R. C., \& Moser, E. (2007). Amygdala activation and facial expressions: explicit emotion discrimination versus implicit emotion processing. Neuropsychologia, 45, 2369-2377. 
Hariri, A. R., Bookheimer, S. Y., \& Mazziotta, J. C. (2000). Modulating emotional responses: effects of a neocortical network on the limbic system. Neuroreport, 11, 43-48.

Hershler, O., Golan, T., Bentin, S., \& Hochstein, S. (2010). The wide window of face detection. Journal of Vision, 10(10), 21, 1-14.

Heutink, J., Brouwer, W. H., de Jong, B. M., \& Bouma, A. (2011). Conscious and unconscious processing of fear after right amygdala damage: A single case ERP-study. Neurocase, $17,297-312$

Johnson, M. H. (2005) Subcortical face processing. Nature Reviews Neuroscience, 6, 766-774.

Kanwisher, N. (2001) Faces and places: Of central (and peripheral) interest. Nature Neuroscience, 4, 455-456.

Kanwisher, N., McDermott, J., \& Chun, M. M. (1997). The fusiform face area: A module in human extrastriate cortex specialized for face perception. Journal of Neuroscience, 17, $4302-4311$

Kim, M. J., \& Whalen, P. J. (2009). The structural integrity of an amygdala-prefrontal pathway predicts trait anxiety. Journal of Neuroscience, 29(37), 11614-11618.

Lange, K., Williams, L. M., Young, A. W., Bullmore, E. T., Brammer, M. J., Williams, S. C. R., Gray, J. A., et al. (2003). Task instructions modulate neural responses to fearful facial expressions. Biological Psychiatry, 53(3), 226-232.

LeDoux, J. \& Phelps, E. (2008). Emotional networks in the brain. In M. Lewis, J. HavilandJones \& L. Barrett (Eds.), Handbook of emotions (pp. 159 - 179). New York, NY: Guilford Press.

Levy, I., Hasson, U., Avidan, G., Hendler, T., \& Malach, R. (2001). Center-periphery organization of human object areas. Nature Neuroscience, 4, 533-539. 
Liu, L., \& Ioannides, A. A. (2010). Emotion separation is completed early and it depends on visual field presentation. PLoS ONE, 5(3), 14.

Lori, N. F., Akbudak, E., Shimony, J. S., Cull, T. S., Snyder, A. Z., Guillory, R. K., \& Conturo, T. E. (2002). Diffusion tensor fiber tracking of human brain connectivity: aquisition methods, reliability analysis and biological results. NMR in Biomedicine, 15(7-8), 494515.

Luo, Q., Holroyd, T., Jones, M., Hendler, T., \& Blair, J. (2007). Neural dynamics for facial threat processing as revealed by gamma band synchronization using MEG. NeuroImage, $34,839-847$

McDonald, A. J. (1998). Cortical pathways to the mammalian amygdala. Progress in Neurobiology, 55(3), 257-332.

Morawetz, C., Baudewig, J., Treue, S., \& Dechent, P. (2010). Diverting attention suppresses human amygdala responses to faces. Frontiers in Human Neuroscience, 4.

Morawetz, C., Baudewig, J., Treue, S., \& Dechent, P. (2011). Effects of spatial frequency and location of fearful faces on human amygdala activity. Brain Research, 1371, 87-99.

Mormann, F., Dubois, J., Kornblith, S., Milosavljevic, M., Cerf, M., Ison, M., Tsuchiya, N., Kraskov, A., Quiroga, R. Q., Adolphs, R., Fried, I., \& Koch, C. (2011). A categoryspecific response to animals in the right human amygdala. Nature Neuroscience, 14, 1247-1249.

Morris, J. S., Öhman, A., \& Dolan, R. J. (1999). A subcortical pathway to the right amygdala mediating "unseen" fear. Proceedings of the National Academy of Sciences of the United States of America, 96, 1680-1685. 
O'Doherty, J. P. (2004). Reward representations and reward-related learning in the human brain: insights from neuroimaging. Current Opinion in Neurobiology, 14(6), 769-776.

Öhman, A. (2005). The role of the amygdala in human fear: Automatic detection of threat. Psychoneuroendocrinology, 30(10), 953-958.

Öhman, A. (2009). Of snakes and faces: An evolutionary perspective on the psychology of fear. Scandinavian Journal of Psychology, 50, 543-552.

Padmala, S., Lim, S.L., \& Pessoa, L. (2010). Pulvinar and affective significance: Responses track moment-to-moment stimulus visibility. Frontiers in Human Neuroscience, 4, 64.

Palermo, R., \& Rhodes, G. (2007). Are you always on my mind? A review of how face perception and attention interact. Neuropsychologia, 45(1), 75-92.

Pessoa, L., \& Adolphs, R. (2010). Emotion processing and the amygdala: from a 'low road' to 'many roads' of evaluating biological significance. Nature Reviews Neuroscience, 11, $773-782$

Pessoa, L., McKenna, M., Gutierrez, E., \& Ungerleider, L.G. (2002). Neural processing of emotional faces requires attention. Proceedings of the National Academy of Sciences of the United States of America, 99, 11458-11463.

Phelps, E.A., O’Connor, K.J., Gatenby, J.C., Gore, J.C., Grillon, C., \& Davis, M. (2001). Activation of the left amygdala to a cognitive representation of fear, Nature Neuroscience, 4, 437-441.

Preibisch, C., Lanfermann, H., Wallenhorst, T., Walter, H., \& Erk, S. (2009). Neuroanatomical correlates of visual field bias: a sensitive system for detecting potential threats? Brain Research, 1263, 69-77. 
Provost, F., \& Fawcett, T. (1997). Analysis and visualization of classifier performance: comparison under imprecise class and cost distributions. Science And Technology, 43-48.

Rauch, S. L., Whalen, P. J., Savage, C. R., Curran, T., Kendrick, A., Brown, H. D., Bush, G., Breiter, H. C., \& Rosen, B. R. (1997). Striatal recruitment during an implicit sequence learning task as measured by functional magnetic resonance imaging. Human Brain Mapping, 5, 124-132.

Rolls, E. T. (2007). The representation of information about faces in the temporal and frontal lobes. Neuropsychologia, 45, 124-143.

Ruge, H., \& Wolfensteller, U. (2010). Rapid formation of pragmatic rule representations in the human brain during instruction-based learning. Cerebral Cortex, 20, 1656-1667.

Saint-Cyr, J. A., Ungerleider, L. G., \& Desimone, R. (1990). Organization of visual cortical inputs to the striatum and subsequent outputs to the pallido-nigral complex in the monkey. Journal of Comparative Neurology, 298(2), 129-156.

Sander, D., Grafman, J., \& Zalla, T. (2003). The human amygdala: an evolved system for relevance detection. Reviews in the Neurosciences, 14, 303-316.

Sprengelmeyer, R., Young, A. W., Sprengelmeyer, A., Calder, A. J., Rowland, D., Perrett, D., Homberg, V., \& Lange, H. (1997). Recognition of facial expressions: Selective impairment of specific emotions in Huntington's disease. Cognitive Neuropsychology, $14,839-879$

Stanislaw, H., \& Todorov, N. (1999). Calculation of signal detection theory measures. Behavior research methods instruments computers a journal of the Psychonomic Society Inc, $31(1), 137-149$ 
Stefanacci, L., \& Amaral, D. G. (2002). Some observations on cortical inputs to the macaque monkey amygdala: an anterograde tracing study. Journal of Comparative Neurology, $451(4), 301-323$

Strasburger, H., \& Jüttner, M. (2011). Peripheral vision and pattern recognition: A review. Journal of Vision, 11(5), 1-82.

Sugiura, M., Kawashima, R., Nakamura, K., Okada, E., Kato, T., Nakamura, A., Hatano, K., Itoh, K., Kojima, S., \& Fukuda, H. (2000). Passive and active recognition of one's own face. Neuroimage, 11, 36-48.

Tamietto, M., \& de Gelder, B. (2010). Neural bases of the non-conscious perception of emotional signals. Nature Reviews Neuroscience, 11, 697-709.

Tamietto, M., Pullens, P., Gelder, B. de, Weiskrantz, L., \& Goebel, R. (2012). Subcortical connections to human amygdala and their changes following destruction of the visual cortex. Current Biology, 22(15), 1449-1455.

Taylor, S. F., Phan, K. L., Decker, L. R., \& Liberzon, I. (2003). Subjective rating of emotionally salient stimuli modulates neural activity. Neuroimage, 18, 650-659.

Thorpe, S. J., Gegenfurtner, K. R., Fabre-Thorpe, M., \& Bülthoff, H. H. (2001). Detection of animals in natural images using far peripheral vision. European Journal of Neuroscience, 14(5), 869-876.

Tong, F., Nakayama, N., Moscovitch, M., Weinrib, O., \& Kanwisher, N. (2000). Response properties of the human fusiform face area. Cognitive Neuropsychology. 17 (1) 257-279.

van Asselen, M., Júlio, F., Januário, C., Campos, E. B., Almeida, I., Cavaco, S., \& CasteloBranco, M. (2012). Scanning patterns of faces do not explain impaired emotion 
recognition in Huntington disease: Evidence for a high level mechanism. Frontiers in Psychology, 3, 31 .

Vuilleumier, P., Armony, J. L., Driver, J., \& Dolan, R. J. (2003). Distinct spatial frequency sensitivities for processing faces and emotional expressions. Nature Neuroscience, 6 , 624-631.

Whalen, P. J., Shin, L. M., McInerney, S. C., Fischer, H., Wright, C. I., \& Rauch, S. L. (2001). A functional MRI study of human amygdala responses to facial expressions of fear versus anger. Emotion Washington Dc, 1(1), 70-83.

Whalen, P.J., Davis, F.C., Oler, J.A., Kim, H., Kim, M.J., \& Neta, M. (2009). Human amygdala responses to facial expressions of emotion. In P.J. Whalen \& E.A. Phelps (Eds). The Human Amygdala (pp. 265-288). New York: Guilford Press.

Wilson, F.A.W., \& Rolss, E.T. (1993). The effects of stimulus novelty and familiarity on neuronal activity in the amygdala of monkeys performing recognition memory tasks. Experimental Brain Research, 93, 367-382.

Winston, J. S., O’Doherty, J., \& Dolan, R. J. (2003). Common and distinct neural responses during direct and incidental processing of multiple facial emotions. NeuroImage, 20(1), 84-97. 


\section{Captions}

Table 1 - Summary of random-effects (RFX)-GLM contrasts, outputs and statistics for the whole brain analysis.

Fig. 1. Experimental design (slow event related paradigm; stimulus duration: 150 ms; ITI: 7500, 10,000 or 12,500 ms). Examples of left, central and right hemifield presentations using three types of categories: neutral (control nonthreatening) and threatening animal faces, and natural scenes/landscapes without animal categories.

Fig. 2. Effect of spatial location: responses of the amygdala for centrally presented stimuli are larger than for left peripheral presentations in a task dependent manner. The left amygdala is preferentially involved during the implicit threat animal face recognition (task 1), while the right amygdala is more engaged during the explicit threat detection task (task 2). ROI RFX-GLM contrasts: mean differences in parameter estimates (z-normalized beta-values) for the contrasts centre > left, task 1 (A) and 2 (B) are displayed. Legend: amy L, amygdala left; amy R, amygdala right; ${ }^{*}<<0.05$. The bars display the standard error of the mean (SE).

Fig. 3. Effect of stimulus type: responses of the amygdala to the threatening animal faces are larger than to the non-facial stimulus. The left amygdala is preferentially involved in the discrimination of threatening and non-threatening stimuli during the animal face recognition (task 1). ROI RFX-GLM contrasts: mean differences in parameter estimates (z-normalized beta-values) for the contrasts threatening faces $>$ non-threatening faces, task 1 (A) and 2 (B) are displayed. 
Legend: amy L, amygdala left; amy $R$, amygdala right; npo0.05. The bars display the standard error of the mean (SE).

Fig. 4. Interaction effect of stimulus type X spatial location: responses of the (right) amygdala to both threatening and non-threatening animal faces are larger than to the non-facial stimulus, and occur only for centrally presented stimuli during the explicit threat detection task (task 2). ROI RFX-GLM contrasts: z-normalized parameter estimates (beta-values) for the contrasts 'centre: threatening faces $>$ non-faces' and 'centre: non-threatening faces $>$ non-faces', task 2, are displayed. ${ }^{*} p<0.01$ and ${ }^{* *} p<0.005$. The bars display the standard error of the mean (SE).

Fig. 5. Activated regions yielded by the RFX group analysis for the contrast explicit threat detection task (task 2) > implicit threat animal face recognition (task 1). Cluster threshold correction was set at $p<0.01$ with a minimum cluster size of 15 voxels.

Fig. 6. Two different striatal regions are differentially activated depending on task type and spatial location: (top, (A)) central processing recruits the right caudate, (bottom, (B)) peripheral processing recruits the left putamen. Peak voxel coordinates (TAL) are displayed. Cluster threshold correction was set at $p<0.01$ with a minimum cluster size of 15 voxels, for peripheral comparisons, (B), and of 13, for central ones, $(A)$. 


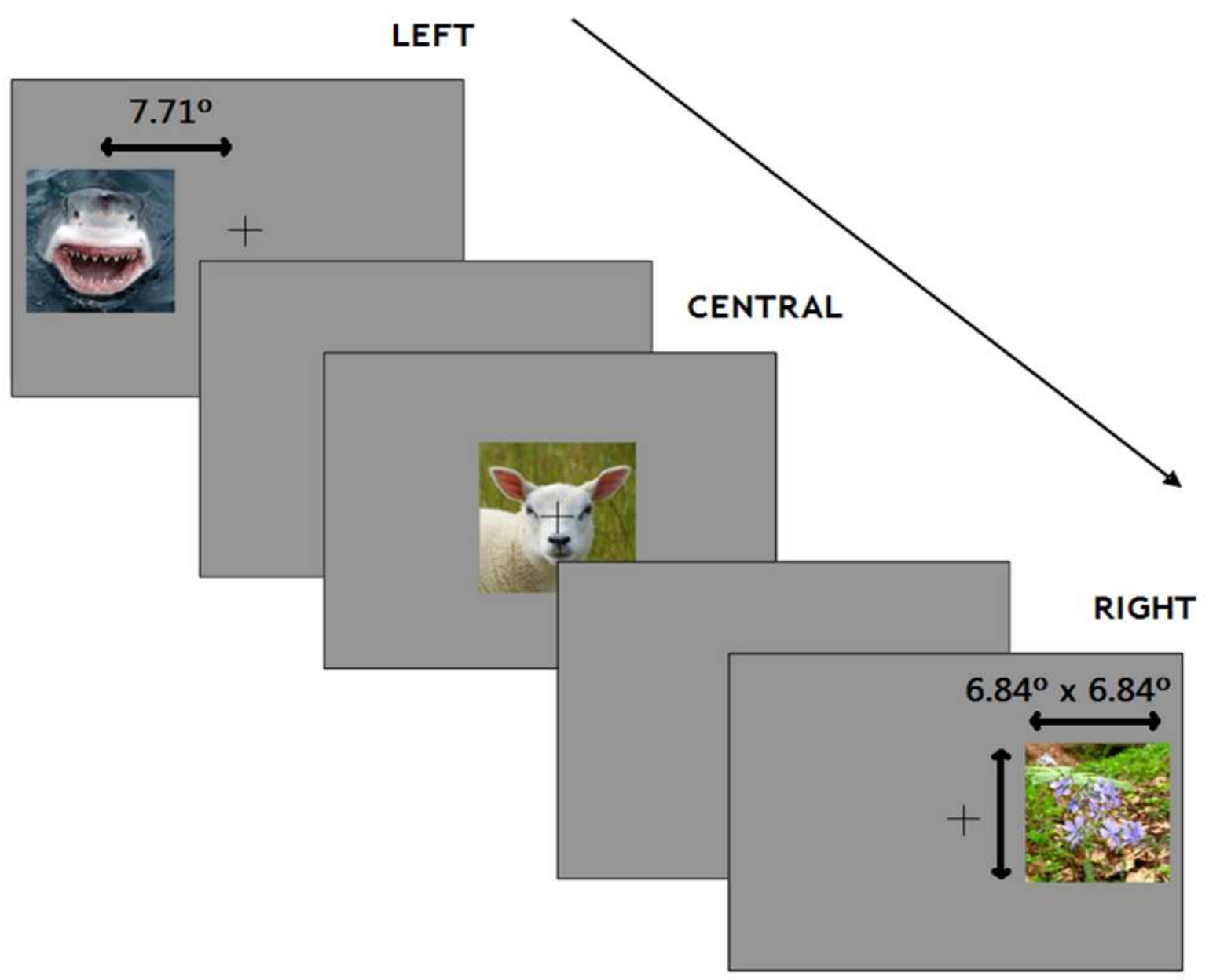

Fig. 1. 


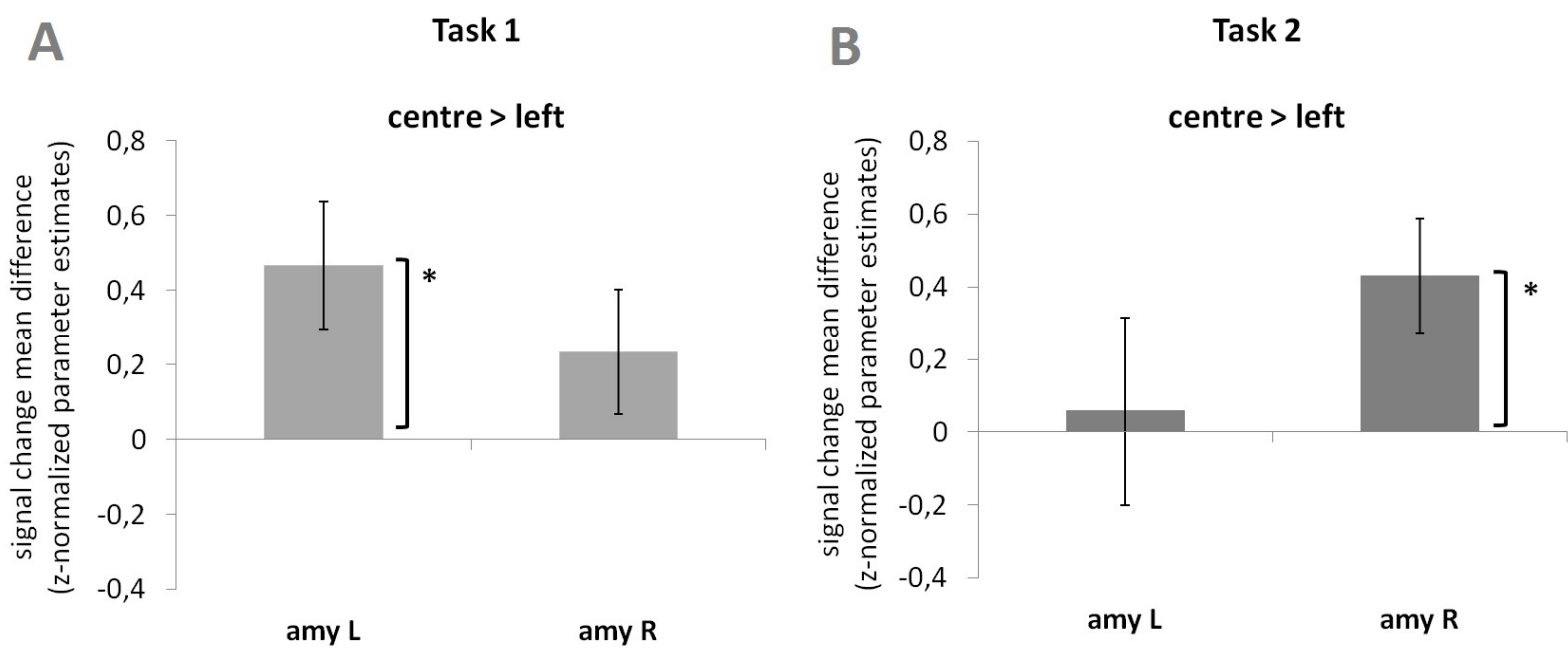

Fig. 2. 
A

Task 1

threatening faces > control non-faces

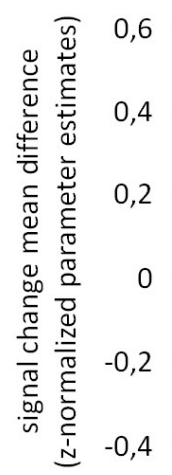

B

Task 2

threatening faces > control non-faces

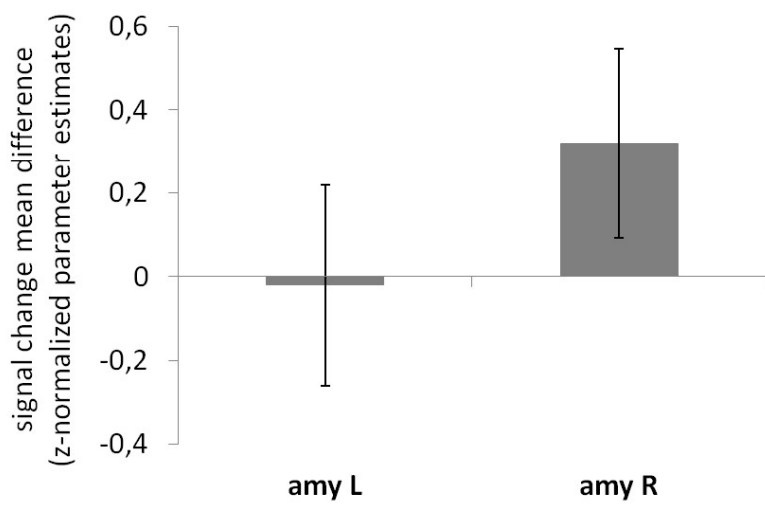

Fig. 3. 
Task 2

spatial location $x$ stimulus type (right amygdala)

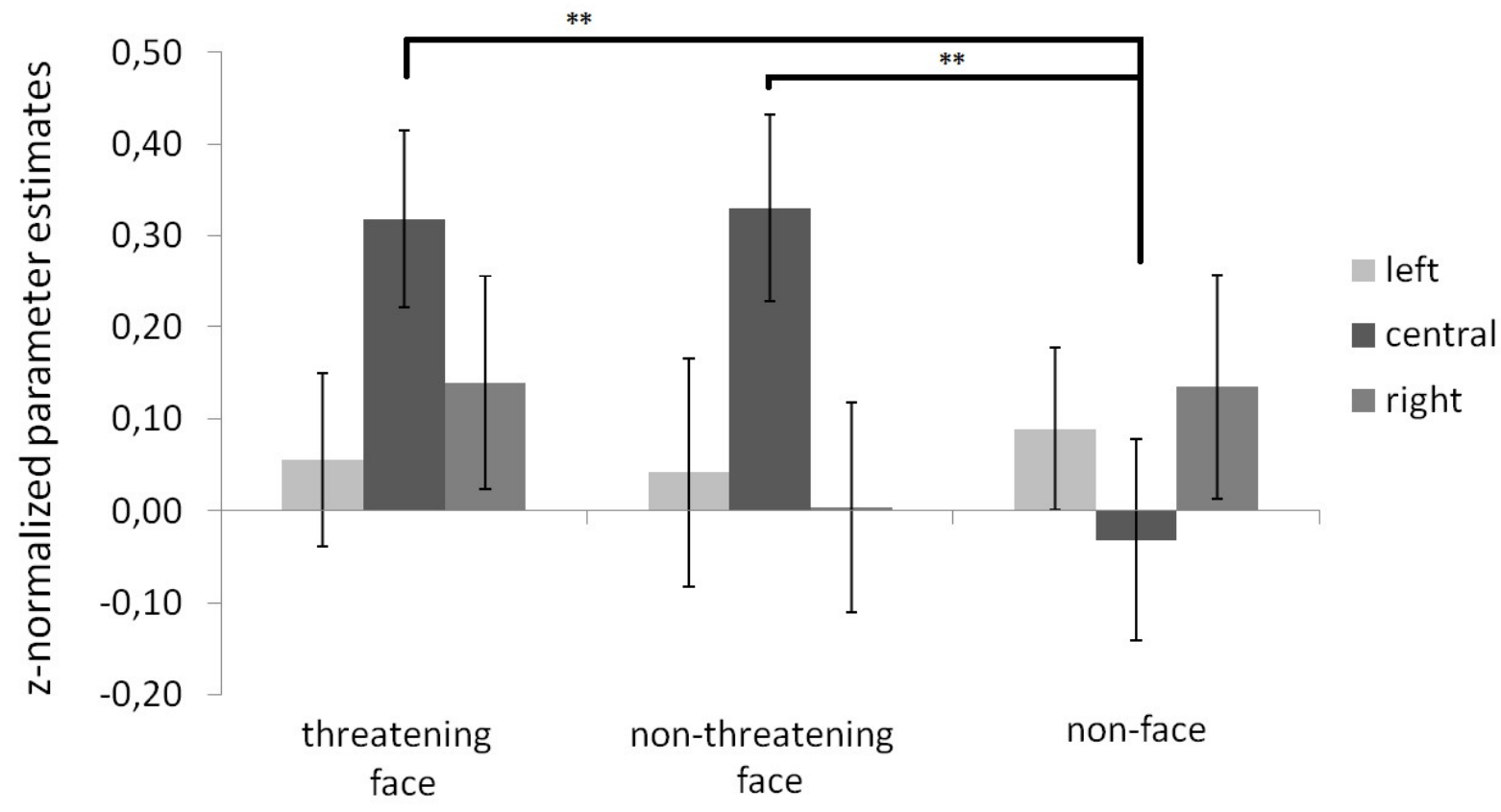

Fig. 4. 


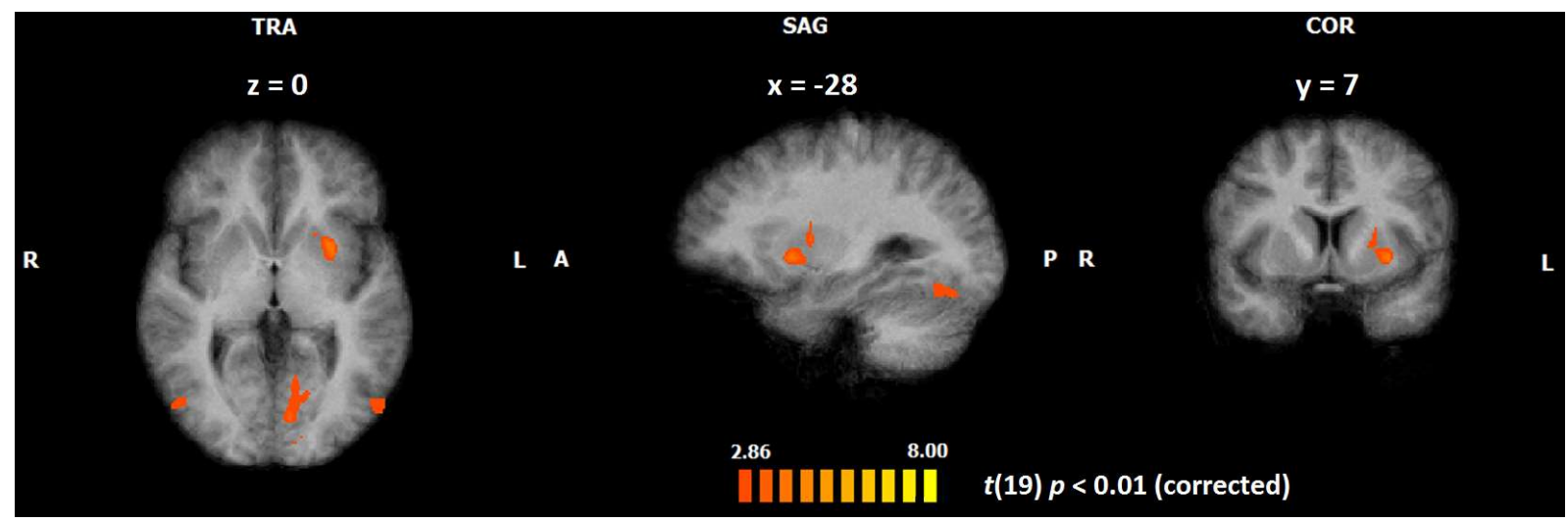

Fig. 5. 


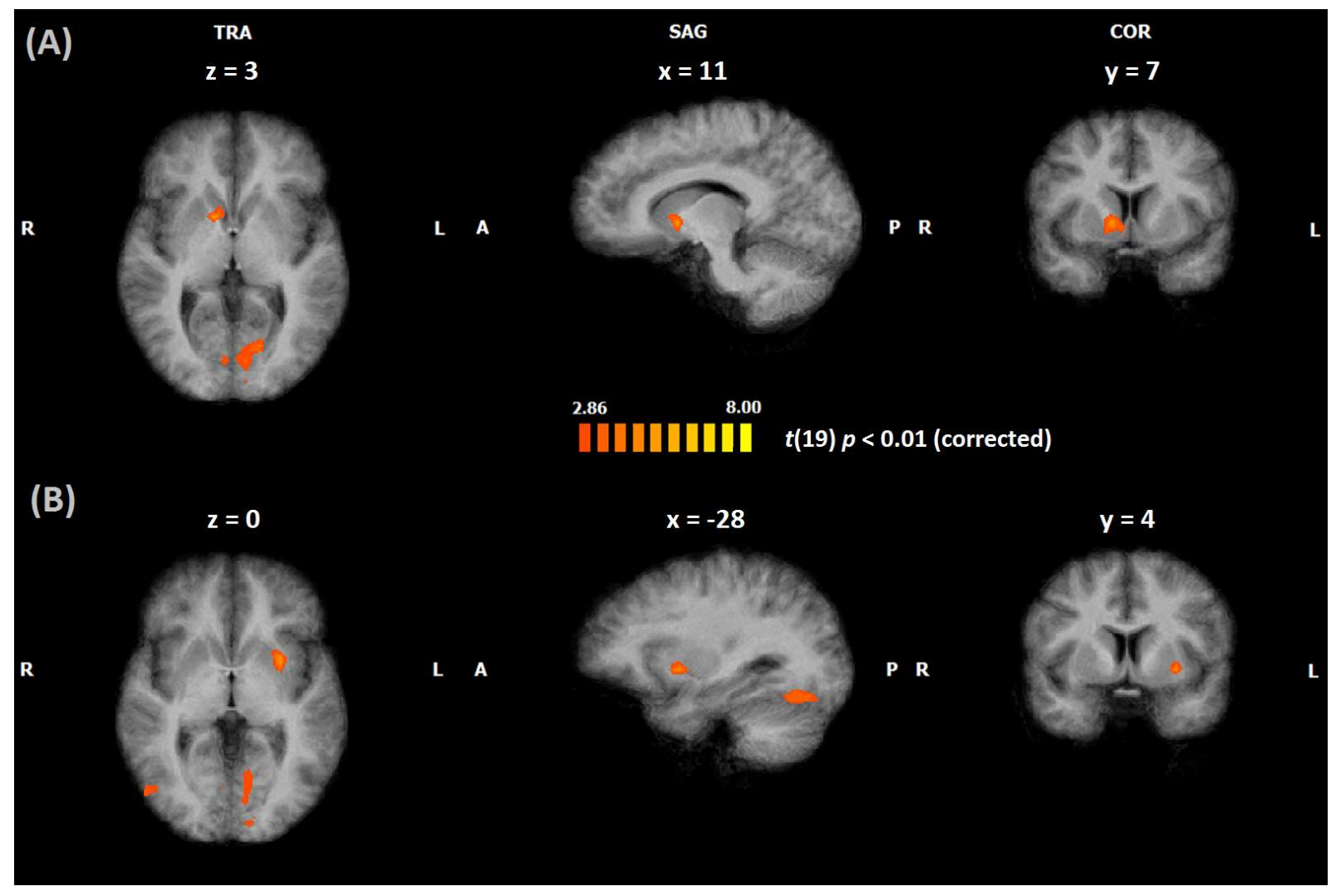

Fig. 6. 
Table 1

Whole brain analyses: summary of random-effects (RFX)-GLM contrasts, outputs and statistics

\begin{tabular}{|c|c|c|c|c|c|c|}
\hline Region & $\begin{array}{l}\text { Peak X } \\
(\mathrm{TAL})\end{array}$ & $\begin{array}{l}\text { Peak Y } \\
\text { (TAL) }\end{array}$ & $\begin{array}{l}\text { Peak Z } \\
\text { (TAL) }\end{array}$ & Nr of voxels & $t$ & $p$ \\
\hline \multicolumn{7}{|c|}{ Contrast a : explicit threat detection $>$ implicit threat animal face recognition } \\
\hline r occipital fusiform gyrus & 38.0 & -71.0 & -9.0 & 806 & 4.809 & 0.000122 \\
\hline $\mathrm{r}$ cuneus & 14.0 & -77.0 & 15.0 & 429 & 3.577 & 0.002012 \\
\hline 1 lingual gyrus & -7.0 & -77.0 & 3.0 & 2248 & 4.458 & 0.000270 \\
\hline 1 medial frontal gyrus & -10.0 & 55.0 & 15.0 & 467 & 6.045 & 0.000008 \\
\hline 1 putamen & -28.0 & 7.0 & 0.0 & 851 & 4.817 & 0.000120 \\
\hline 1 cerebellum & -28.0 & -62.0 & -18.0 & 456 & 4.050 & 0.000683 \\
\hline 1 middle temporal gyrus & -55.0 & -68.0 & 12.0 & 535 & 3.948 & 0.000863 \\
\hline \multicolumn{7}{|c|}{ Contrast $b$ : centre $>$ periphery } \\
\hline r lateral occipital gyrus & 23.0 & -89.0 & -12.0 & 59312 & 8.625 & 0.000000 \\
\hline$r$ posterior cingulate gyrus & 20.0 & -14.0 & 42.0 & 921 & -6.331 & 0.000004 \\
\hline I superior frontal gyrus & -19.0 & 46.0 & 39.0 & 779 & 3.886 & 0.000994 \\
\hline I middle temporal gyrus & -40.0 & -74.0 & 24.0 & 1640 & 4.602 & 0.000195 \\
\hline
\end{tabular}

Contrast c : centre: explicit threat detection > implicit threat animal face recognition

$\begin{array}{lcccccc}\mathrm{r} \text { caudate head } & 11.0 & 7.0 & 3.0 & 426 & 5.312 & 0.000040 \\ 1 \text { lingual gyrus } & -10.0 & -77.0 & 6.0 & 1126 & 4.492 & 0.000250\end{array}$

Contrast $d$ : periphery: explicit threat detection $>$ implicit threat animal face recognition

$\begin{array}{lcccccc}\text { r occipital fusiform gyrus } & 38.0 & -71.0 & -9.0 & 721 & 5.523 & 0.000025 \\ \text { r cerebellum } & 20.0 & -62.0 & -12.0 & 420 & 3.868 & 0.001036 \\ \text { r posterior cingulate gyrus } & 2.0 & -68.0 & 12.0 & 3428 & 4.369 & 0.000330 \\ \text { I putamen } & -28.0 & 4.0 & 0.0 & 485 & 5.291 & 0.000042 \\ \text { I cerebellum } & -25.0 & -71.0 & -18.0 & 741 & 4.252 & 0.000431\end{array}$

All contrasts were performed at $p<.01$ using cluster threshold correction. X, Y and Z represent Talairach coordinates. $r$, right; $l$, left. 


\section{Highlights}

1. The right amygdala responds preferentially to faces presented at central locations.

2. The left amygdala responds to threatening faces in implicit face recognition tasks.

3. Central processing of explicit threat in the caudate nucleus.

4. Peripheral processing of threat dominates in the putamen. 
Graphical Abstract:

ROI-based analysis: amygdala

spatial location

ROI-based analysis: amygdala

spatial location $\mathrm{x}$ stimulus type

Whole brain analysis: basal ganglia

task type

x spatial location
Task 1

Task 2
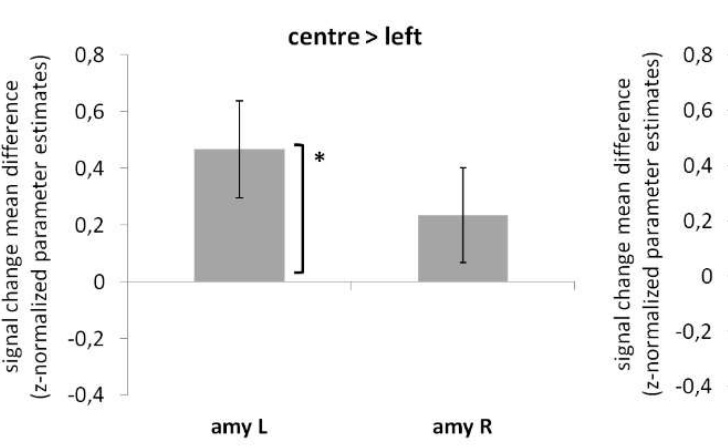

centre $>$ left
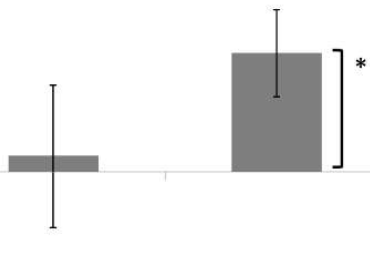

task 2: spatial location $x$ stimulus type (right amygdala)
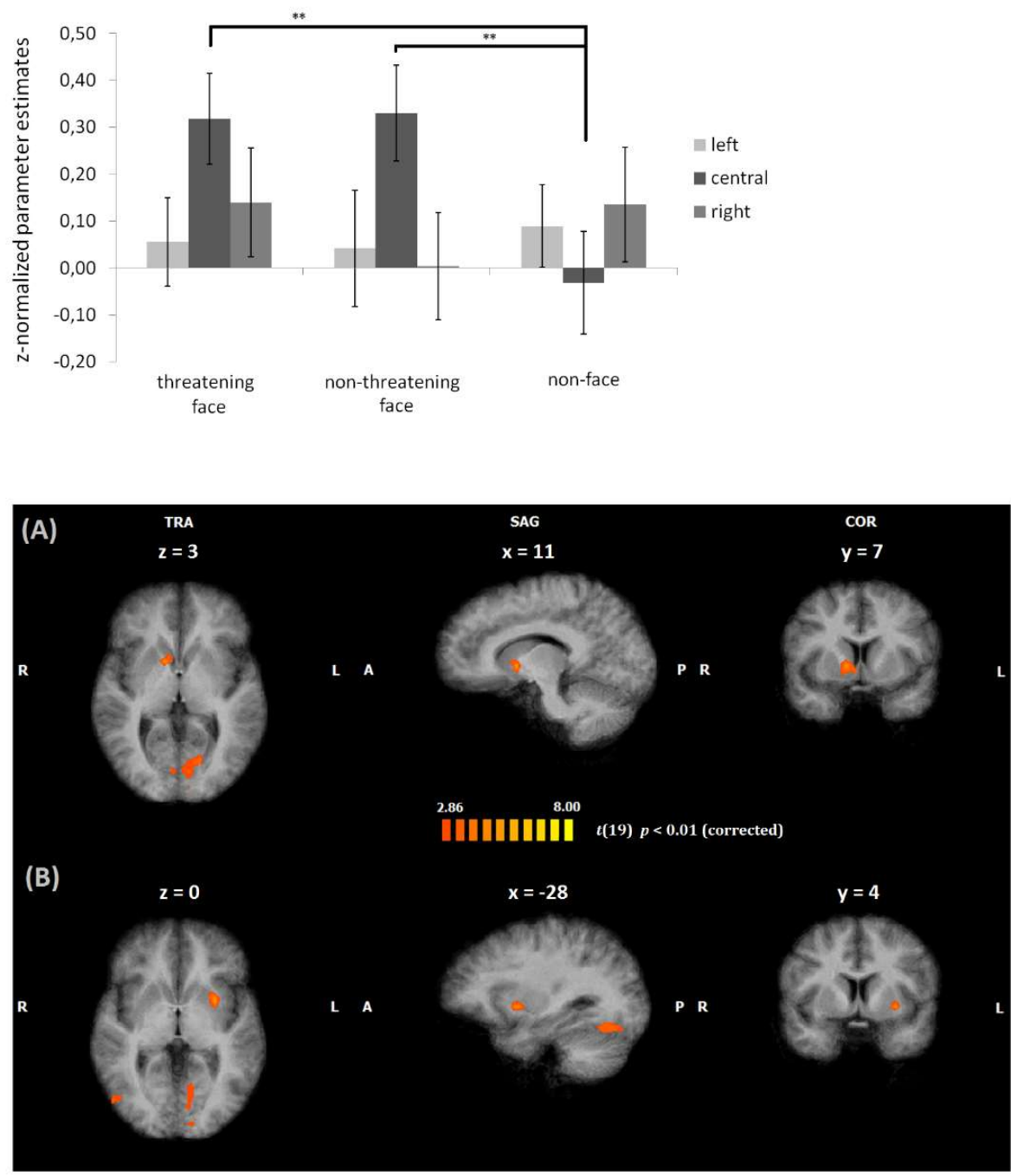